OPEN ACCESS

Edited by:

Rick Friedman,

University of California, San Diego,

United States

Reviewed by:

Oliver Adunka,

The Ohio State University,

United States

Andrea Canale,

University of Turin, Italy

${ }^{*}$ Correspondence:

Sebastián A. Ausill

s.ausili@miami.edu

Specialty section:

This article was submitted to

Neuro-Otology

a section of the journa

Frontiers in Neurology

Received: 01 June 2020

Accepted: 16 July 2020

Published: 30 September 2020

Citation:

Ausili SA, Agterberg MJH, Engel A,

Voelter C, Thomas JP, Brill $S$,

Snik AFM, Dazert S, Van Opstal AJ

and Mylanus EAM (2020) Spatial Hearing by Bilateral Cochlear Implant

Users With Temporal Fine-Structure

Processing. Front. Neurol. 11:915.

doi: 10.3389/fneur.2020.00915

\section{Spatial Hearing by Bilateral Cochlear Implant Users With Temporal Fine-Structure Processing}

\author{
Sebastián A. Ausili ${ }^{1,2 *}$, Martijn J. H. Agterberg ${ }^{1,3}$, Andreas Engel ${ }^{4}$, Christiane Voelter ${ }^{4}$, \\ Jan Peter Thomas ${ }^{4}$, Stefan Brill ${ }^{5}$, Ad F. M. Snik ${ }^{1}$, Stefan Dazert ${ }^{4}$, A. John Van Opstal ${ }^{1}$ and \\ Emmanuel A. M. Mylanus ${ }^{3}$ \\ ${ }^{1}$ Department of Biophysics, Radboud University, Donders Institute for Brain, Cognition and Behaviour, Nijmegen, \\ Netherlands, ${ }^{2}$ Department of Otolaryngology, University of Miami, Miami, FL, United States, ${ }^{3}$ Department of \\ Otorhinolaryngology, Radboud University Medical Center, Donders Institute for Brain, Cognition and Behaviour, Nijmegen, \\ Netherlands, ${ }^{4}$ Department of Otorhinolaryngology Head and Neck Surgery, St. Elisabeth-Hospital, Ruhr-University Bochum, \\ Bochum, Germany, ${ }^{5}$ MED-EL, Starnberg, Germany
}

Several studies have demonstrated the advantages of the bilateral vs. unilateral cochlear implantation in listeners with bilateral severe to profound hearing loss. However, it remains unclear to what extent bilaterally implanted listeners have access to binaural cues, e.g., accurate processing of interaural timing differences (ITDs) for low-frequency sounds $(<1.5 \mathrm{kHz})$ and interaural level differences (ILDs) for high frequencies $(>3 \mathrm{kHz})$. We tested 25 adult listeners, bilaterally implanted with MED-EL cochlear implant $(\mathrm{Cl})$ devices, with and without fine-structure (FS) temporal processing as encoding strategy in the low-frequency channels. In order to assess whether the ability to process binaural cues was affected by fine-structure processing, we performed psychophysical ILD and ITD sensitivity measurements and free-field sound localization experiments. We compared the results of the bilaterally implanted listeners with different numbers of FS channels. All Cl listeners demonstrated good sensitivity to ILDs, but relatively poor to ITD cues. Although there was a large variability in performance, some bilateral $\mathrm{Cl}$ users showed remarkably good localization skills. The FS coding strategy for bilateral $\mathrm{Cl}$ hearing did not improve fine-structure ITD processing for spatial hearing on a group level. However, some Cl listeners were able to exploit weakly informative temporal cues to improve their low-frequency spatial perception.

Keywords: sound localization, bilateral cochlear implants, fine-structure, interaural level differences, interaural time differences

\section{INTRODUCTION}

The research performed in bilateral cochlear implant (CI) listeners clearly showed that they outperform users with a unilateral CI. Apart from improved speech understanding in noise (1-5), sound localization performance in the bilateral CI condition also improved when compared to unilateral CI [e.g., $(1,5,6)]$. However, the benefit of bilateral implantation is not equivalent to binaural hearing, and the performance gap between bilateral CI vs. normal-hearing $(\mathrm{NH})$ listeners is still significant $(7,8)$. Normal-hearing listeners localize sounds in azimuth with high acuity and precision, thanks to the efficient processing of interaural level differences (ILDs) and interaural timing differences (ITDs). ILDs are extracted from the high-frequency hearing range $(>3 \mathrm{kHz})$, while ITDs are conveyed in the temporal fine structure of low-frequency sounds $(<1.5 \mathrm{kHz})(9,10)$. 
CI technology mainly aimed at improving speech perception. Thus, most available pulse-encoding strategies [e.g., continuous interleaved sampling, or CIS (11)] stimulate the different electrodes with a fixed pulse frequency, in which the current strength of the pulses is modulated by the sound's envelope over the full applicable frequency range. To potentially improve the temporal representation, MED-EL included a CI pulse stimulation strategy that aimed to preserve the low-frequency temporal fine structure of the acoustic signal (fine-structure processing, or FSP). Their algorithm incorporates a zero-crossing analysis in the low-frequency range, after which the coded electrical signal is conveyed to electrode contacts of the CI (i.e., up to four channels in the low-frequency apical turn or up to about $1 \mathrm{kHz}$ according to the coding configuration).

Studies have suggested that bilateral CI listeners benefit from the FSP stimulation strategy in a speech discrimination test in noise (12-16), especially for native speakers of tonal languages (17-19). Dorman et al. (20) studied sound localization in FSP and CIS bilateral users and did not find differences between subgroups. In addition, they observed that low-frequency sounds were poorly localized compared to high-frequency and broadband sounds, suggesting that the bilateral FSP is not adding a benefit for sound localization. However, it is unclear whether this negative finding resulted from a low number of lowfrequency fine-structure channels since this was not reported. Recently, Eklöf and Tideholm (21) found that half of the CI listeners with FSP had ITD perception within the physiological range (10/20; mean threshold of $330 \pm 250 \mu \mathrm{s})$ compared to none in the group without fine-structure (FS) coding. Although some FSP listeners appeared to have ITD sensitivity, this stimulation protocol did not improve their low-frequency sound localization. However, their dichotic stimuli, used for the ITD perceptual tests (500-ms tone pips of $250 \mathrm{~Hz}$ with rise and fall times of $100 \mathrm{~ms}$ ), were not the same as those presented in the free field for sound localization (1.6-s duration speech-shaped sounds or low-pass-filtered music snippets).

Here, we performed two experiments to test sound localization and ILD/ITD processing in experienced bilateral CI users with and without FSP. In order to quantify the effect of the number of bilateral fine-structure channels, we assessed performance for three subgroups: (i) without FSP; (ii) with two bilateral FSP channels; or (iii) with four bilateral FSP channels. In principle, if FSP improves the ITD perception of bilateral CI users by providing reliable cues (21), a larger number of available FSP channels could increase the potential to exploit this cue. We presented different frequency ranges to dissociate ITD and ILD perception with psychoacoustical tasks and free-field sound localization.

\section{METHODS}

\section{Listeners \\ Cl Users}

Twenty-five bilaterally deaf patients implanted with bilateral CIs (BICI) participated in the experiments. Their ages ranged from 22 to 77 years (53 \pm 16.3 years). In the Netherlands, bilateral CIs in adults is not reimbursed. Therefore, in order to acquire bilaterally implanted adult subjects, cooperation was sought with the ENT Clinic of St. Elisabeth-Hospital of Ruhr-University in Bochum in our neighboring country Germany, where reimbursement of bilateral cochlear implantation for adults has been standard care for years. All included subjects had been implanted and were recruited at the University Clinic of Bochum (Germany) and traveled to Radboud University in Nijmegen (the Netherlands) to be assessed in the sophisticated spatial hearing labs at the Radboud University in Nijmegen. All research protocols and informed consent forms were approved by the Medical Ethical Committee in Bochum prior to the start of the experiments. Table 1 shows the ages at test, ages of implantation for each ear, device type, and coding strategy in use. Most participants suffered from a progressive hearing loss. Three patients suffered from an infection (P8: encephalitis at birth; P11: pneumococcal meningitis; and P12: mumps infection), and in one case the etiology of the hearing loss was not known (P9). All CI subjects had bilateral profound hearing loss, with no residual hearing in low frequencies at the time of implantation. Electrode impedance measures were within the normal range, demonstrating normal functioning of CI. Normal electrically evoked compound action potentials (eCAPs) were obtained intraoperatively for all subjects, suggesting good coupling between the electrodes and neural substrate. All electrode insertions were reported to be complete, and subjects were implanted with the same electrode design and length in both ears. To study the effect of FSP, we grouped the patients according to the number of bilateral fine-structure channels: "FS4" refers to listeners with four (the maximum number) FS channels on both sides $(n=12)$; "FS2" to two FS channels on either side $(n=8)$; and "no-FSP" indicates either no FS channel at all or a non-matched low number (e.g., 0 and 1 on the right and left sides, respectively, $n=5$ ). The latter group was considered as a control group within the CI users.

The fitting was performed under the currently applied standard procedures for bilateral CI programming, where each device is first fit independently. Later, narrow-band noises were presented in free field for right/left loudness balancing and CI users indicated their percept of the mid-sagittal plane. Note that all CI users were very experienced with the tested coding strategy and no major changes were done on their fitting for this particular experiment.

\section{Normal-Hearing Controls}

Eleven NH listeners (ages 24-37 years) were enrolled in the experiments as controls. All had normal thresholds (within 20 $\mathrm{dB}$ of audiometric zero), as determined by a standard pure-tone audiogram (ISO 8253-1:2010). Listeners had no visual and motor disorders and were naive about the purpose of the experiments.

\section{Fine Structure Processing}

Low-frequency sounds are coded both in place and time in the apical region of the cochlea. However, most implants only extract the envelope of the incoming signal for all electrodes (frequency bands), thus eliminating the fine-structure cues. In contrast, FSP developed by MED-EL modifies the timing of pulse stimulation to code temporal information in the low frequencies. 
TABLE 1 | Demographic and general information about subjects.

\begin{tabular}{|c|c|c|c|c|c|c|c|c|c|c|c|}
\hline \multirow[t]{2}{*}{ Subject } & \multirow{2}{*}{$\begin{array}{l}\text { Age at test } \\
\text { (years) }\end{array}$} & \multicolumn{2}{|c|}{ Age of implantation } & \multicolumn{2}{|c|}{ Implant and electrode } & \multicolumn{2}{|c|}{ Audio processor } & \multicolumn{2}{|c|}{ Coding strategy } & \multicolumn{2}{|c|}{ FS channels } \\
\hline & & $\mathbf{R}$ & $\mathbf{L}$ & $\mathbf{R}$ & $\mathbf{L}$ & $\mathbf{R}$ & $\mathbf{L}$ & $\mathbf{R}$ & $\mathbf{L}$ & $\mathbf{R}$ & $\mathbf{L}$ \\
\hline P1 & 77 & 71 & 73 & Concerto Flex28 & Sonata Standard & Sonnet & Sonnet & FS4HR & FS4HR & 4 & 4 \\
\hline P2 & 62 & 58 & 56 & Sonata Standard & Sonata Standard & Opus2 & Opus2 & FSP & FSP & 2 & 2 \\
\hline P3 & 43 & 42 & 39 & Sonata Flex28 & Sonata Flex28 & Opus2 & Sonnet & FS4HR & FS4HR & 4 & 4 \\
\hline P4 & 29 & 28 & 29 & Sonata Flex28 & Sonata Flex28 & Sonnet & Sonnet & FS4HR & FS4HR & 4 & 4 \\
\hline P5 & 71 & 64 & 63 & Sonata Standard & Sonata Standard & Opus2 & Opus2 & FS4HR & FS4HR & 4 & 4 \\
\hline P6 & 76 & 69 & 73 & Sonata Flex28 & Sonata Standard & Opus2 & Opus2 & FS4HR & FS4HR & 4 & 4 \\
\hline P7 & 66 & 62 & 64 & Sonata Flex28 & Sonata Standard & Opus2 & Opus2 & FSP & FSP & 2 & 2 \\
\hline P8 & 22 & 1 & 6 & Pulsar Standard & Combi40 Standard & Sonnet & Sonnet & FSP & CIS & 1 & 0 \\
\hline P9 & 22 & 1 & 5 & Combi40+ Standard & Combi40+ Standard & Opus2 & Opus2 & CIS & CIS & 0 & 0 \\
\hline P10 & 56 & 51 & 52 & Sonata Flex28 & Sonata Flex28 & Opus2 & Opus2 & FS4HR & FS4HR & 4 & 4 \\
\hline P11 & 49 & 44 & 44 & Sonata Standard & Sonata Standard & Sonnet & Sonnet & FSP & FSP & 2 & 2 \\
\hline P12 & 41 & 36 & 39 & Concerto Flex28 & Concerto FlexSoft & Sonnet & Opus2 & FS4HR & FS4HR & 4 & 3 \\
\hline P13 & 62 & 55 & 54 & Sonata Standard & Sonata Standard & Sonnet & Sonnet & FS4HR & FS4HR & 4 & 4 \\
\hline P14 & 46 & 44 & 43 & Concerto Standard & Concerto Medium & Sonnet & Sonnet & CIS & FSP & 0 & 1 \\
\hline P15 & 49 & 46 & 46 & Sonata Flex28 & Sonata Standard & Sonnet & Sonnet & FSP & FSP & 2 & 2 \\
\hline P16 & 57 & 55 & 56 & Synchrony Flex28 & Synchrony Flex28 & Rondo & Rondo & FSP & FSP & 2 & 2 \\
\hline P17 & 55 & 52 & 48 & Sonata Standard & Sonata Flex28 & Opus2 & Opus2 & FSP & FSP & 2 & 1 \\
\hline P18 & 50 & 48 & 47 & Sonata Standard & Sonata FlexSoft & Rondo & Rondo & FSP & FSP & 2 & 2 \\
\hline P19 & 52 & 47 & 45 & Sonata Standard & Sonata Standard & Opus2 & Opus2 & FS4HR & FS4HR & 4 & 4 \\
\hline P20 & 70 & 63 & 65 & Sonata Flex28 & Sonata Standard & Opus2 & Opus2 & FSP & FSP & 2 & 2 \\
\hline P21 & 76 & 71 & 70 & Sonata Flex28 & Sonata Flex28 & Opus2 & Opus2 & FS4HR & FS4HR & 4 & 4 \\
\hline P22 & 26 & 18 & 24 & Sonata Flex28 & Sonata Standard & Sonnet & Sonnet & FS4HR & FS4HR & 4 & 4 \\
\hline P23 & 67 & 58 & 62 & Concerto FlexSoft & Sonata Standard & Opus2 & Sonnet & FSP & FSP & 2 & 2 \\
\hline P24 & 50 & 47 & 40 & Sonata Standard & Sonata Flex28 & Opus2 & Rondo & FS4HR & FS4HR & 4 & 4 \\
\hline P25 & 50 & 35 & 36 & Combi40+ Standard & Combi40+ Standard & Sonnet & Sonnet & CIS & CIS & 0 & 0 \\
\hline
\end{tabular}

FSP coding is identical to the CIS-based stimulation (11), except lower-frequency channels, where channel-specific sampling sequences (CSSS) replace the fixed fast-rate pulse train. The CSSS are a series of pulses triggered by the positive zero crossings in the filter's output signals, which are then modulated in amplitude by the extracted envelope of the corresponding channel.

Based on this initial stimulation protocol, the first version of the FS coding strategy was introduced in 2006. The number of apical channels with CSSS varied from one to three according to the fitting variables for a given CI user. Later, FS4 was developed, ensuring CSSS channels up for the first four apical channels (up to $1 \mathrm{kHz}$ ). Finally, FS4-P was released, which allows parallel time coding on the four apical CSSS channels. In our study, and as mentioned before, we adopted our own nomenclature to define the FS coding strategy referring to the bilateral number of CSSS or FS channels (see previous section).

Normally, in bilateral CI fittings, the audio processors are not synchronized with each other. However, the apical electrodes on the FSP strategy are locked to the zero crossing of the acoustic input. Since the acoustic input is highly correlated (or "synchronized") between ears, theoretically, the pulses can be delivered preserving ITDs. However, the sampling rate is the limiting factor for a proper zero-crossing representation. For the
CSSS channels, it is typically situated between 3 and $10 \mathrm{kHz}$, which can lead to temporal accuracies of $0-333$ and $0-100 \mu \mathrm{s}$, respectively (22). In bilateral FSP configurations, this procedure results in an interaural jitter in the order of \pm 100 to $\pm 333 \mu \mathrm{s}$, which can perturb ITD perception.

\section{ILD and ITD Perception \\ Stimuli and Task}

Psychometric experiments were used to measure the ILD and ITD sensitivity of the listeners, together with their potential side bias. The stimuli were generated in MATLAB (The MathWorks Inc., Natick, MA, USA), played through an external sound card (MOTU Ultralite, Cambridge, MA). For bilateral CI users, the stimuli were delivered through the CI processor's audio input (only bypassing their microphones), and $\mathrm{NH}$ was tested via headphones (Beyerdynamic, DT 770 Pro, Heilbronn, Germany). Tasks were designed as two-alternative forced choice (2AFC). After the sound was presented, the listener had to respond by pressing the left or right arrow on a keyboard, indicating the perceived side relative to the mid-sagittal plane of the head.

For ILD sensitivity testing, the level between the two inputs was changed, while the ITD was fixed at $0 \mathrm{~s}$. During the test, the acoustic power of the signal was kept constant, maintaining the same overall loudness for all ILD magnitudes. This task 


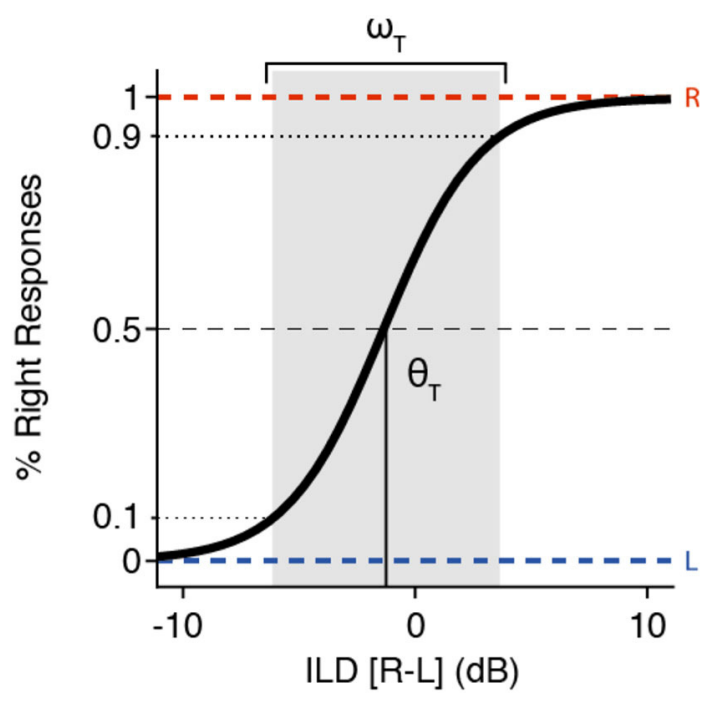

FIGURE 1 | Example of an interaural level difference (ILD) psychometric curve, described by Equation (1), with its two parameters indicated. The shaded zone indicates the sensitivity range, $\omega \mathrm{T}$ (here, $\sim 10 \mathrm{~dB}$ ) around the threshold, $\theta_{\mathrm{T}}$ (here taken at $-1.25 \mathrm{~dB}$ ).

was evaluated with two frequency ranges: low-pass (LP, 0.15$0.8 \mathrm{kHz}$ ), generally covering the four apical channels, and highpass (HP, $1.5-10 \mathrm{kHz}$ ), where medial and basal electrodes are stimulated (electrodes 5-12). The applied ILDs for the BICI users ranged from +10 to $-10 \mathrm{~dB}$ and were divided into 16 equal steps of $1.25 \mathrm{~dB}$. Each ILD was randomly tested 15 times along the experiment, making a total of 240 trials. The $\mathrm{NH}$ listeners were tested over a narrower ILD range between $\pm 5 \mathrm{~dB}$ (divided into 16 equal steps of $0.625 \mathrm{~dB}$ ); in addition, the +10 - and $-10-\mathrm{dB}$ sounds were presented to obtain the two extreme data points. Also, each ILD was evaluated 15 times, making a total of 270 trials for this group.

For ITD testing, the ILD was kept constant at $0 \mathrm{~dB}$, while the onset time between the ears was systematically varied. For this task, LP $(0.15-0.8 \mathrm{kHz})$ noise bursts were presented. ITDs were varied between $\pm 2 \mathrm{~ms}$ for BICI users (in 16 equal 0.25 - $\mathrm{ms}$ steps) and $\pm 0.2 \mathrm{~ms}$ for $\mathrm{NH}$ listeners (in 16 equal 0.025 -ms steps), together with +0.8 and $-0.8 \mathrm{~ms}$ as the extreme data points. The same amount of trials as for the ILD task was performed.

\section{Data Analysis}

To describe the psychophysical ILD and ITD data, we performed a sigmoid fit over the binary left/right responses with the following logistic function (23) (Figure 1):

$$
p\left(x_{T}\right)=\left(1+\mathrm{e}^{-4.39 \frac{\left(x_{\mathrm{T}}-\theta_{\mathrm{T}}\right)}{\omega_{\mathrm{T}}}}\right)^{-1}
$$

with $x_{\mathrm{T}}$ the acoustic cue of the target [ILD (in decibels) or ITD (in milliseconds)] and $\theta_{\mathrm{T}}$ the perceptual bias (the same unit as $x_{\mathrm{T}}$ ). Positive/negative values reflect a left/rightward bias, and the function is point-symmetric around $\theta_{\mathrm{T}}$ (in decibels or milliseconds). The listener's sensitivity to the cue is described by the width, $\omega_{\mathrm{T}}$ (in decibels or milliseconds), which, in the present parametrization (Equation 1), denotes the 10-90\% width of the sigmoid (see Figure 1, expressed in the same units as the stimulus). Note that the width and sensitivity are inversely related: a larger width yield, less sensitivity.

To interpret the ILD and ITD sensitivity, we extracted physiological ranges from a $5^{\circ}$ horizontal resolution impulse response library (24). The maximum ILD/ITD value computed as a function of azimuth is used in our analysis and referred to as the physiological limit.

\section{Sound Localization Setup and Stimuli}

Sound localization performance was tested for broadband (0.15$10 \mathrm{kHz})$, high-pass $(1.5-10 \mathrm{kHz})$, and low-pass $(0.15-0.8 \mathrm{kHz})$ noise bursts of $150 \mathrm{~ms}$. As in the psychophysical tasks, the frequency ranges were selected to cover apical (LP; channels 1-4), medial-basal (HP; channels 5-12), and the complete electrode array stimulation (BB; channels 1-12). Sound levels were presented at 50,60, and $70 \mathrm{dBA}$ and target locations were distributed over the two-dimensional frontal space, between $\pm 75^{\circ}$ in azimuth and $\pm 30^{\circ}$ in elevation. Stimuli were presented in a dark, anechoic room as described by Van Bentum et al. (25). The subjects were asked to localize these noise bursts by pointing with a rapid head movement to the perceived location of the stimulus.

\section{Experiment}

Each sound localization trial started with the presentation of a green fixation LED at straight ahead $\left(0^{\circ}\right.$ azimuth and $0^{\circ}$ elevation). Using a head-fixed laser pointer, the subjects were instructed to align the laser dot with the fixation LED to ensure the same head orientation at the start of each trial. After the subject pressed a button, the fixation light was turned off within 100-300 ms, followed by the target sound with a 200-ms delay. The subjects were asked to point the laser dot as fast and accurately as possible toward the perceived sound location. The acquisition time of the head movement was $2.5 \mathrm{~s}$.

\section{Data Analysis}

Head movements were detected automatically from the calibrated head position signals using a custom-made Matlab script that checked for head velocities exceeding $20 \%$ s. Onset and offset of the head movements were detected by the program and visually checked off-line.

The target-response relationship of the BICI users was modeled with a sigmoid (Figure 2) using the following equation:

$$
\alpha_{R}=90 \cdot c \cdot \tanh \left(\frac{g \cdot\left(\alpha_{T}-b_{T}\right)}{90}\right)+b_{R}
$$

$\alpha_{T}$ and $\alpha_{R}$ correspond to the target and response angles (in degrees), respectively. The range $\left(\Delta_{\text {LOC }}\right)$, or compression, of the localization response is quantified by $c$ (dimensionless), e.g., if $c$ is 0.5 , the responses are constrained between $\pm 45^{\circ}$ in azimuth. The slope of the sigmoid is determined by $\mathrm{c}$ and $g$ (dimensionless). Finally, parameters $b_{\mathrm{T}}$ and $b_{\mathrm{R}}$ correspond to the target and response biases (in degrees), respectively. The first derivative of 


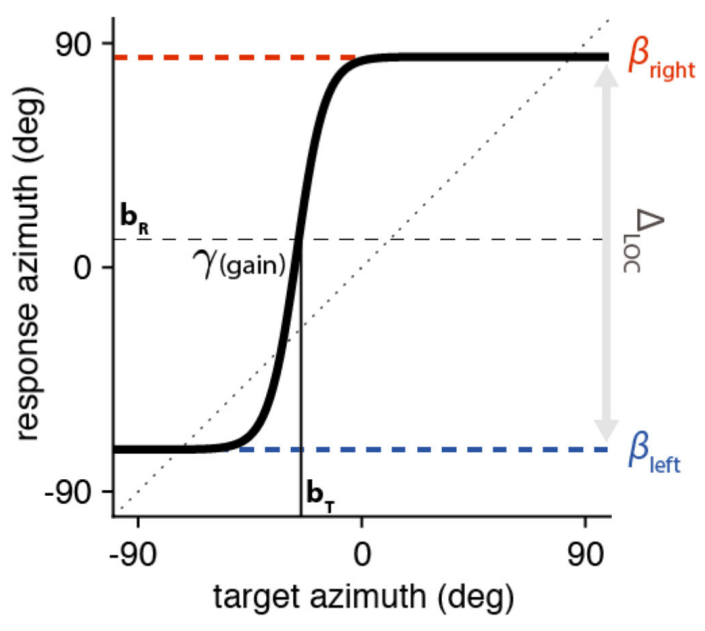

FIGURE 2 | Sound localization fitting example as described by Equation (3). The sigmoid is centered around the target where the curve is equidistant from the two asymptotes (in the example, $b_{T}=-24^{\circ}$ (leading to a rightward response bias) and $b_{\mathrm{R}}=+9^{\circ}$, respectively). The maximal localization gain, or $\gamma$, is calculated at the target bias location $\left(\alpha_{T}=b_{T}\right.$; in this example, $\left.\gamma=4.9\right)$. The asymptotes $\left(\beta_{\text {left }}=-73^{\circ}\right.$ and $\left.\beta_{\text {right }}=+84^{\circ}\right)$ define the range of the localization response $\left(\Delta_{\mathrm{LOC}}=157^{\circ}\right)$.

the sigmoid at $\alpha_{T}=b_{T}$ provides the maximum slope of the fit (i.e., the maximum localization gain), $\gamma$, which is an interaction of the compression of the response and the sigmoid gain:

$$
\gamma=c \cdot g
$$

The limits of the response range (in degrees) are determined by the asymptotes of the fit and are referred to as $\beta_{\text {left }}=b_{\mathrm{r}}$ - 90c and $\beta_{\text {right }}=b_{\mathrm{r}}+90 c$, for the leftward and rightward limits, respectively. Note that a perfect localization response would result in $\gamma=1$ and $b_{\mathrm{T}}=b_{\mathrm{R}}=0^{\circ}$, with $\beta_{\text {left }} \leq 90^{\circ}$ and $\beta_{\text {right }}>90^{\circ}$. A gain that far exceeds $1(\gamma>>1)$ suggests a tendency toward left/right discrimination (what we here refer to as lateralization performance); in an extreme case, the sigmoid fit would resemble a step function, showing only left/right localization (extreme lateralization).

To obtain an overall measure for the response accuracy, we also computed the mean absolute error (MAE) across trials, according to:

$$
\mathrm{MAE}=\frac{1}{\mathrm{~N}} \sum_{\mathrm{n}=1}^{\mathrm{N}}\left|\alpha_{\mathrm{R}}^{\mathrm{n}}-\alpha_{\mathrm{T}}^{\mathrm{n}}\right|
$$

with $\alpha_{R}$ the response azimuth (in degrees), $\alpha_{T}$ the target azimuth (in degrees), and $N$ the number of trials. Note that, in our experiments and setup configuration, extreme lateralization performance (i.e., if $\alpha_{T} \geq 0^{\circ}, \alpha_{R}=+75^{\circ}$, and for $\alpha_{T}<0^{\circ}$, $\alpha_{R}=-75^{\circ}$ ), would result in a MAE $=37^{\circ}$.

\section{Statistical Analysis}

For sound localization analysis, separate $N$-way ANOVAs were performed with as independent factors-subject (random), presentation level, stimulus bandwidth, and FSP groupand as dependent variables $-b_{\mathrm{T}}$ (target bias), $b_{\mathrm{R}}$ (response bias), $c$ (compression factor of the response range), $\gamma$ (gain), and MAE.

Means and the 95\% confidence intervals of the dependent variables are also reported in the Results ( \pm 2 times the standard error of the mean). This confidence interval was also used to calculate the statistical significance between means. All analyses were performed with MATLAB software.

\section{RESULTS}

\section{ILD and ITD Perception}

To determine the sensitivity of ILDs and ITDs, we use $\omega$ as a measure of cue sensitivity and $\theta$ to quantify the right/left bias (see Methods). The stimuli were LP and HP noises, addressing the apical and medial-basal electrodes for CI users, respectively. In general, FSP cochlear implant listeners revealed some, albeit poor, ITD sensitivity. All subjects had their sensitivity range $(\omega)$ beyond the physiological range (see gray area in Figure 3), where this cue will saturate under free-field hearing. However, most ( $n=22$ out of 25) participants were able to detect high-frequency ILDs within a usable range $(<20 \mathrm{~dB})$. Both tasks showed considerable variability among the CI subjects in sensitivity $(\omega)$ and threshold $(\theta)$.

To illustrate the data analysis and responses for individual cases, we show the results from five representative BICI listeners with zero, two, and four FSP channels (Figures 3A-O) as well as for a $\mathrm{NH}$ control listener, for comparison (Figures 3P-R). The sensitivity of the ITD cue for the BICI users ranged from $1.7 \mathrm{~ms}$ (FS2 listener P2; Figure 3F) to $9.6 \mathrm{~ms}$ (no FS listener P19; Figure 3L), while the NH example showed a $\omega_{\text {ITD }}$ that was much smaller, at $0.2 \mathrm{~ms}$ (NH3; Figure 3O). Although the modest sensitivity to ITD is insufficient to correctly lateralize the sound, it may nevertheless have offered a weakly informative cue for the listeners to be perceived (e.g., see Figures 3F,L).

In contrast, the ILD sensitivity for the BICI users and the $\mathrm{NH}$ example, quantified by $\omega_{\text {ILD }}$, were more comparable. While P19 performed more poorly with a sensitivity of $\omega_{\mathrm{ILD}}=31.8 \mathrm{~dB}$ for $\mathrm{HP}$ and $\omega_{\mathrm{ILD}}=30.4 \mathrm{~dB}$ for LP, listener $\mathrm{P} 2$ yielded $\omega_{\mathrm{ILD}}=7.9$ $\mathrm{dB}$ and $\omega_{\mathrm{ILD}}=5.8 \mathrm{~dB}$ for $\mathrm{HP}$ and LP sounds, respectively. These examples illustrate the large variability across listeners, but indicate also that most of them had a well-defined sensitivity to this cue. The threshold value, $\theta_{\mathrm{ILD}}$, which characterizes the balance between the right and left ears, varied across listeners. Note that the LP value for $\theta_{\text {ILD }}$ is correlated to $\theta_{\text {ITD }}$ as the ITD was measured at $0 \mathrm{~dB}$ ILD for the same frequency range for all CI listeners $\left(r^{2}=0.59, p<0.001\right)$. For example, $\mathrm{P} 1$ had a rightward ILD bias for LP stimuli with $\theta_{\text {ILD }}=-5.2 \mathrm{~dB}$ and also a negative ITD bias of $\theta_{\text {ITD }}=-1.4 \mathrm{~ms}$ for the same sounds. Also, listeners P9 and P19 showed a consistent relation between the LP-ILD and ITD tasks, but although they yielded smaller ILD thresholds than P1, their ITD thresholds were more extreme to the right than for P1.

An overview of the sensitivity $(\omega)$ and bias $(\theta)$ for all sound types and subgroups is provided in Figure 4. The vertical gray line in Figures $\mathbf{4 A - C}$ illustrates the maximum ILD/ITD for each 


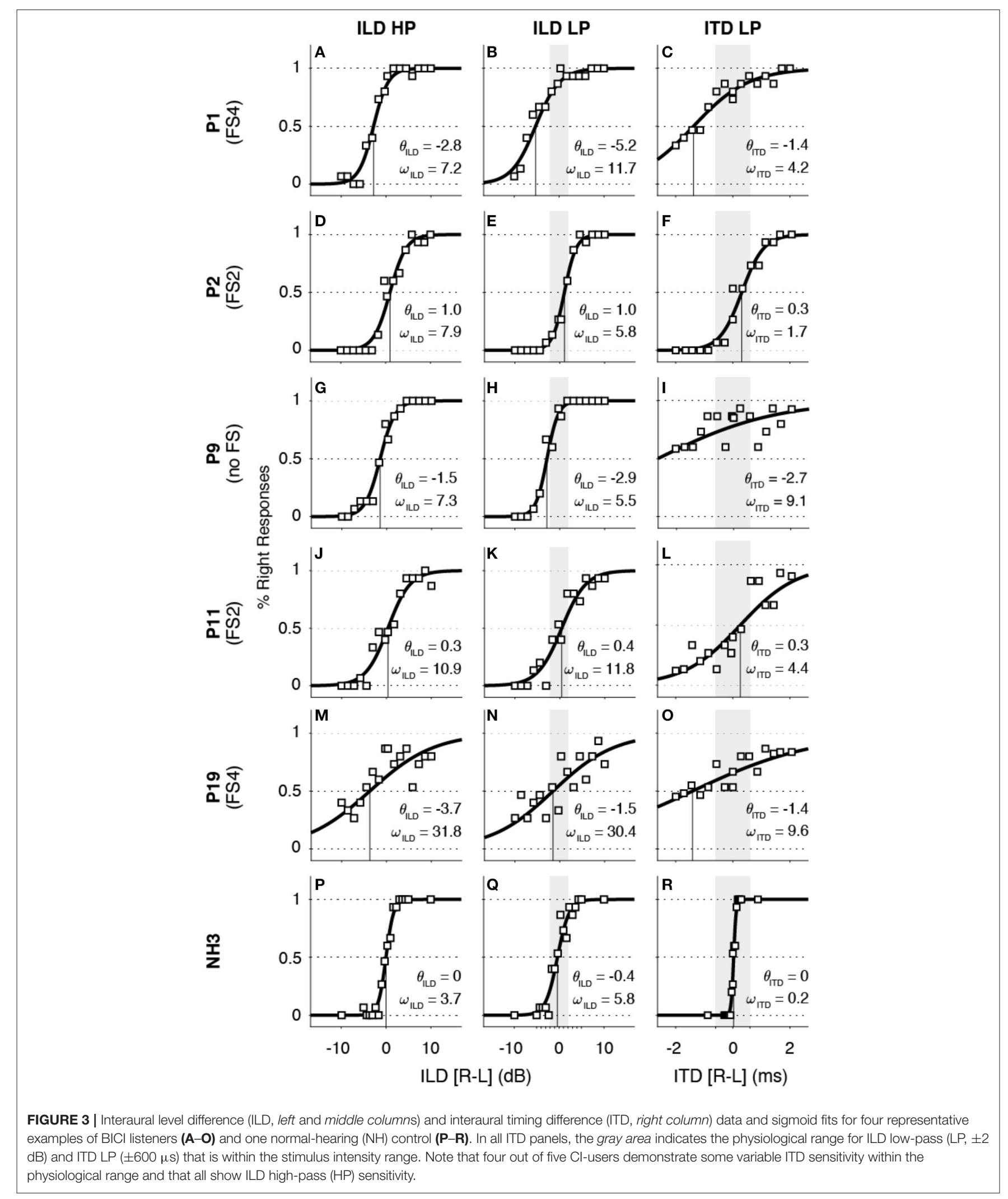

bandwidth (see Methods). We did not observe an effect of the different FS subgroups on ILD or ITD sensitivity (confidence intervals of the means were overlapping). When pooling all groups to compute the overall mean (gray shadowed area in Figure 4), all BICI users yielded $\omega_{\text {ITD }}$ beyond the physiological limit (mean of $5.5 \pm 1.7 \mathrm{~ms}$; Figure $4 \mathrm{C}$ ), which reflects the 

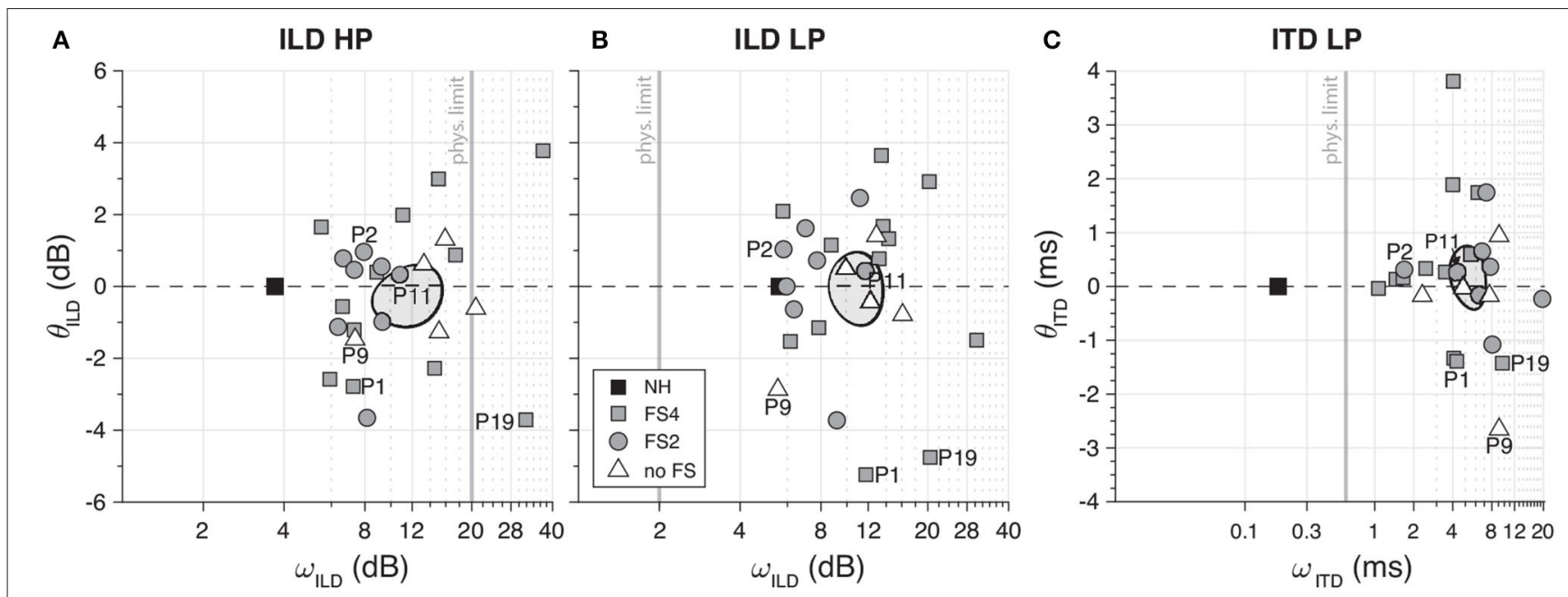

FIGURE 4 | Overall bias $(\theta)$ and sensitivity $(\omega)$ of interaural level difference (ILD) high-pass (A) and low-pass (B) and interaural timing difference (ITD) low-pass (C). Vertical gray lines represent the cue's physiological range values. In all panels, the mean and variability ( $95 \%$ confidence interval) of the data of BICl users are represented by ellipses. As reference, the mean of the $\mathrm{NH}$ results is marked with a gray square. Note that for ILD and ITD low-pass, all CI users fell outside the boundaries ( $2 \mathrm{~dB}$ and $600 \mu \mathrm{s}$, respectively) indicated by the physiological range. Normal-hearing (NH) controls, however, demonstrated clear ITD LP perception.

difficulty to use ITD cues in the free field. Note, however, that some FS subjects demonstrated better ITD sensitivity (around $1 \mathrm{~ms}$ ), which is not far from the physiological limit and, although not sufficient for veridical lateralization, could still serve as a weak but informative cue for azimuth (e.g., P2; Figure 3F). Similarly, the sensitivity for low-frequency ILDs fell outside its small physiological range $( \pm 2 \mathrm{~dB})$ for all CI listeners (mean $=$ $11.2 \pm 2.6 \mathrm{~dB}$; Figure 4B). However, most listeners demonstrated ILD sensitivity for HP (mean $=11.9 \pm 3.5 \mathrm{~dB}$; Figure 4A) and LP sounds (mean $=11.2 \pm 2.6 \mathrm{~dB}$; Figure 4B). All normal-hearing controls showed a high sensitivity to ILD in HP (mean of 3.7 $\pm 1.7 \mathrm{~dB}$; Figure 4A) and ITD in LP (mean of $0.12 \pm 0.02 \mathrm{~ms}$; Figure 4C). It is noteworthy that, in normal hearing subjects, the reduced sensitivity to weak low-pass ILDs is overcome with the ITD perception. There was considerable variability in the biases across BICI listeners, which could reflect that one device was programmed slightly louder than the other. Although the overall means for $\theta_{\mathrm{ILD}}(-0.2 \pm 0.9 \mathrm{~dB}$ for $\mathrm{HP}$ and -0.1 \pm 1.0 for LP; Figures $\mathbf{4 A , B}$, respectively) and $\theta_{\text {ITD }}(0.2 \pm$ $0.6 \mathrm{~ms}$; Figure 4C) did not differ from zero, some subjects had a clear right or left tendency. All NH listeners showed balanced responses for ILD $(0 \pm 0.3 \mathrm{~dB}$ for $\mathrm{HP}$ and $0 \pm 0.2$ for $\mathrm{LP}$; Figures 4A,B, respectively) and ITD ( $0.10 \pm 0.02 \mathrm{~ms}$; Figure 4C), as expected.

\section{Sound Localization}

Localization performance in the free anechoic field was tested with $\mathrm{BB}, \mathrm{HP}$, and LP sounds presented in pseudorandom order at one of the three presentation levels $(50,60$, or $70 \mathrm{~dB}$ SPL) in the two-dimensional frontal hemifield. The presentation levels did not affect the response gain, $\gamma\left(F_{d f}=2=0.8, p=0.5\right)$, the perceived target range, $c\left(F_{d f}=2=1.9, p=0.1\right)$, target bias, $b_{\mathrm{T}}\left(F_{d f=2}=1.6, p=0.2\right)$, or response bias, $b_{\mathrm{R}}\left(F_{d f=2}=1.1\right.$, $p=0.3$ ). Therefore, we pooled all data across levels and analyzed the differences on the three stimulus bandwidths.

To illustrate the overall type of responses from the BICI listeners and the sigmoid fit analysis, Figure 5 shows the results of the same subjects as on the ILD/ITD tasks. Subject P1 localized BB sounds with a good near-linear stimulus-response relation with $\gamma=1.5$ and practically no bias $\left(b_{\mathrm{R}}=1.0^{\circ}\right.$ and $b_{\mathrm{T}}=1.0^{\circ}$; Figure 5A). Moreover, the difference in the $\mathrm{BB}$ and HP performance (Figures 5A,B) suggests that the subtle lowfrequency cues may have been useful and beneficial for this listener. However, the localization performance for LP sounds yielded noisier responses with a higher gain $(\gamma=30.4)$, a higher $\operatorname{MAE}\left(25.9^{\circ}\right)$, and a pronounced rightward bias $\left(t=-43^{\circ}\right.$; Figure 5C).

Listener P2 is an extreme example of sound lateralization as the responses were directed to the far left and far right, irrespective of the stimulus type presented (Figures 5D-F). This listener had a clear leftward bias for the LP stimuli, in line with a positive target bias $\left(b_{\mathrm{T}}=22^{\circ}\right)$. The extreme lateralization performance yielded high MAEs (mean $\mathrm{MAE}=35^{\circ}$ for the three sound types, which is closer to the actual theoretical lateralization value of $37^{\circ}$ ). P9 generated responses to fixed locations, around $\pm 60^{\circ}$, in the left and right hemifields (Figures $5 \mathrm{H}, \mathbf{I}$ ). In this case, the responses were slightly more variable, but with MAEs that were substantially smaller than for P2.

Listeners P11 and P19 showed similar systematic stimulusresponse relations for azimuth than did $\mathrm{P} 1$. In both cases, the central range $\left( \pm 40^{\circ}\right)$ showed an almost linear target-response relationship, but saturating at the edges. Interestingly, both listeners also yielded systematic localization responses for the LP stimuli, with small MAEs and near-normal gains, albeit with a reduced response range $\left(\Delta_{\mathrm{LOC}}=\sim 90-95^{\circ}\right.$; Figures $\left.5 \mathbf{L}, \mathbf{O}\right)$.

To quantify the sound localization performance of all listeners, Figure 6 shows the localization gain $(\gamma)$ against the response 


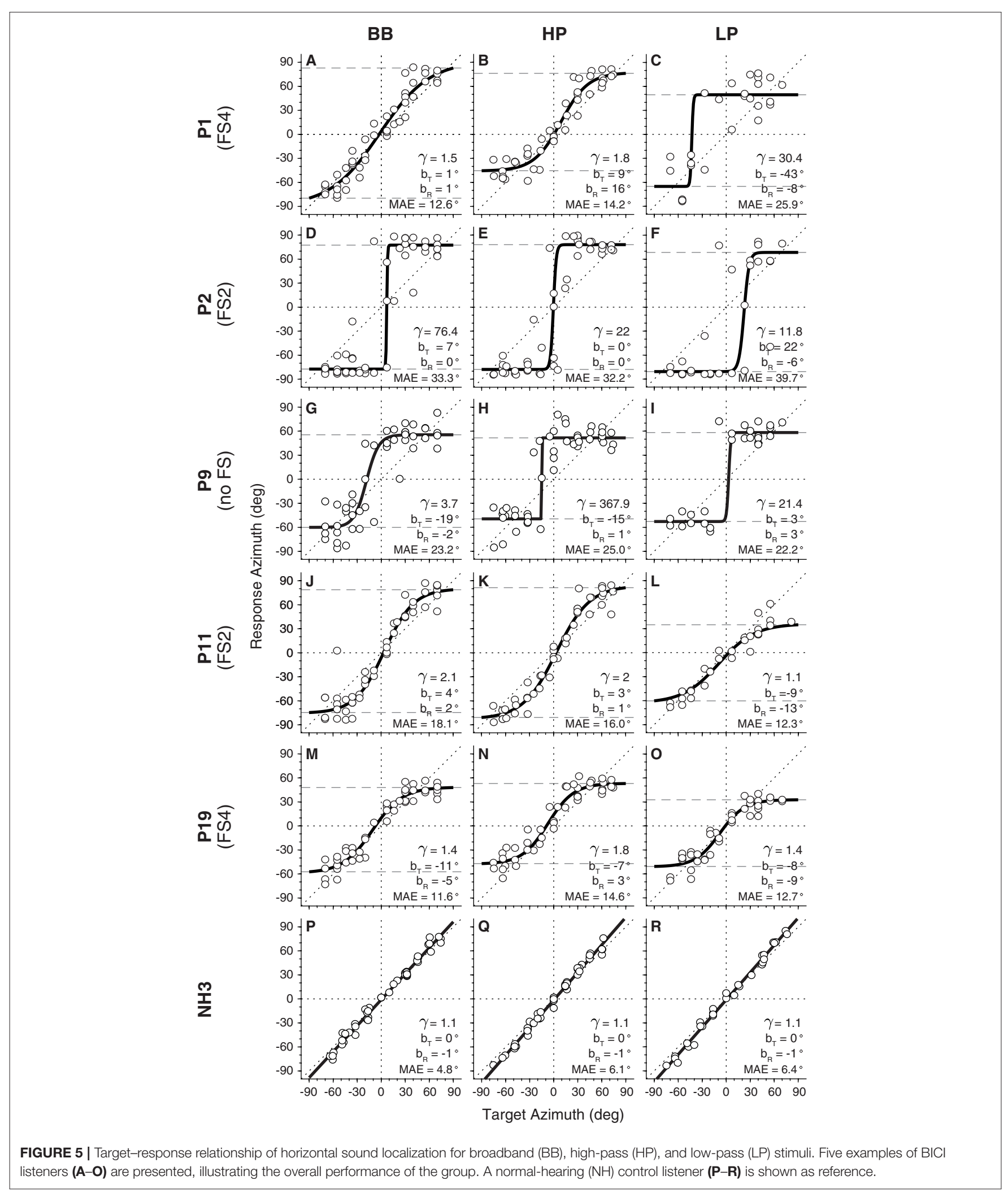

compression ( $c$, also represented as $\left.\Delta_{\mathrm{LOC}}\right)$. Due to the lack of significant differences on $\gamma\left(F_{d f}=2=0.49, p=0.6\right)$ and $c\left(F_{d f}=2=0.59, p=0.559\right)$ between the three groups, we calculated the overall mean per CI group for each stimulus type. Generally, BICI listeners yielded localization gains $>1$ for $\mathrm{BB}$ sounds (mean $=8.3 \pm 6.5$, median $=3.4$; Figure $6 \mathrm{~A}$ ), for 

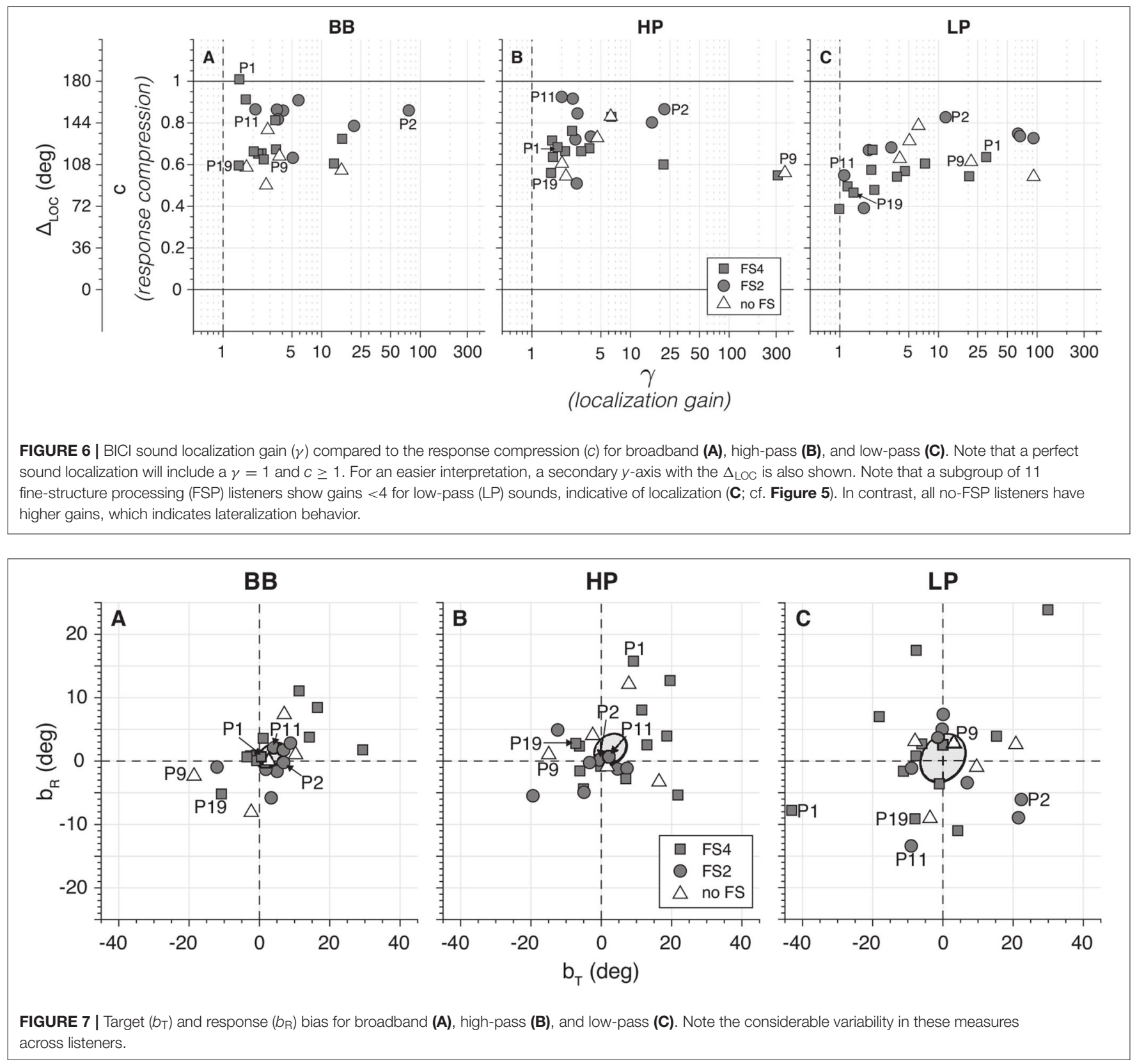

HP stimuli (mean $=33 \pm 40$, median $=2.8$; Figure 6B), and for $\mathrm{LP}$ noise $($ mean $=19.3 \pm 12.7$, median $=4.5$; Figure $6 \mathrm{C})$. Interestingly, only listeners with FSP showed gains $\gamma<4(11 / 19)$ for the LP stimuli (Figure 6C), while all five "no FS" listeners yielded higher gains. This suggests that some BICI listeners appeared to benefit from the weak yet informative low-frequency localization cues provided by the FSP protocol. Note also that the response range was reduced for all BICI subjects as the response compression, $c$, was significantly below 1 , with means of $0.73 \pm$ 0.06 for BB (Figure 6A), $0.70 \pm 0.05$ for HP (Figure 6B), and 0.60 \pm 0.05 for LP sounds (Figure 6C). We also observed a stronger reduction of the response range for LP sounds (mean $=109$ $\left.\pm 9^{\circ}\right)$ when compared to the $\mathrm{BB}\left(\right.$ mean $\left.=130 \pm 9^{\circ}\right)$ and $\mathrm{HP}$ $\left(\right.$ mean $\left.=126 \pm 9^{\circ}\right)$ stimuli.

Figure 7 shows the results for the localization stimulus and response biases. Although the overall mean bias for $\mathrm{BB}\left(b_{\mathrm{T}}=\right.$ $\left.3.4 \pm 4.2^{\circ} ; b_{\mathrm{R}}=0.9 \pm 1.8^{\circ}\right)$, HP $\left(b_{\mathrm{T}}=2.6 \pm 4.6^{\circ} ; b_{\mathrm{R}}=\right.$ $\left.1.8 \pm 2.4^{\circ}\right)$, and $\operatorname{LP}\left(b_{\mathrm{T}}=0.02 \pm 6.5^{\circ} ; b_{\mathrm{R}}=0.4 \pm 3.7^{\circ}\right) \mathrm{did}$ not differ from zero, there was considerable variability across participants, evidencing some remaining perceptual asymmetries between sides.

As an overall measure for the localization performance of the bilateral CI groups, we computed the MAE for the different sounds (Figure 8). Overall, the MAE of the CI listeners was 
around $15^{\circ}$ higher than the $\mathrm{NH}$ performance, but performance was much better than for pure lateralization. BB and HP yielded a similar result, with $21 \pm 3^{\circ}$ and $22 \pm 3^{\circ}$, respectively. LP sounds yielded slightly higher MAEs, with a mean of $24 \pm 3^{\circ}$. The NH controls performed with low MAEs across frequencies, with an overall mean of $7 \pm 1^{\circ}$.

\section{ILD and Free-Field Sound Localization Bias}

The left/right bias was quantified for both psychophysical experiments (as $\theta$ for the ILD/ITD tasks and $b_{\mathrm{T}, \mathrm{R}}$ for sound localization, respectively). As representative measures, we here

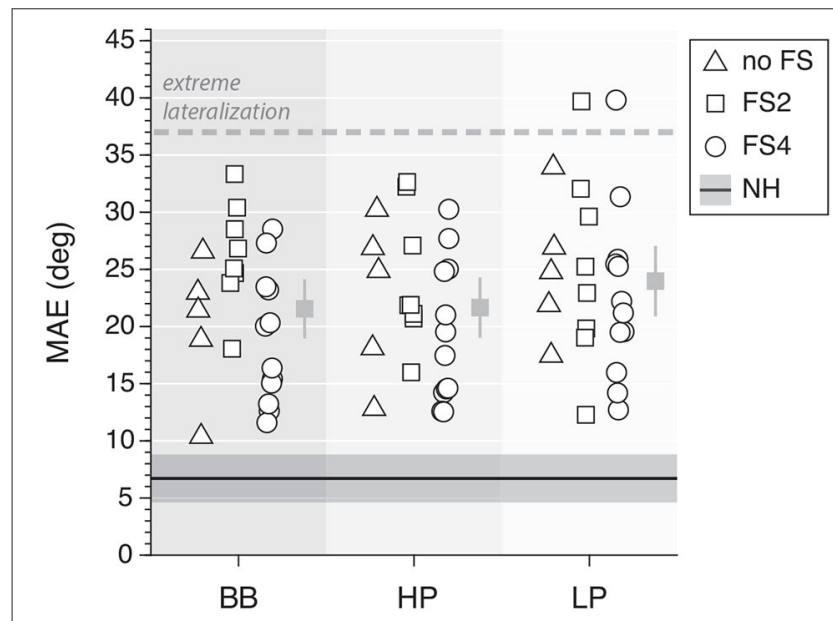

FIGURE 8 | Mean absolute error of sound localization performance for all frequency ranges. The dashed line indicates the theoretical extreme for a pure lateralizer and the dark solid line represents the mean and 95\% confidence interval for the normal-hearing controls. The mean and its 95\% confidence interval for $\mathrm{Cl}$ users are indicated per type of noise (gray squares). present $\theta_{\mathrm{ILD}}$ and $b_{\mathrm{T}}$ for the HP and LP stimuli. We wondered whether the right or leftward free-field target localization bias, $b_{\mathrm{T}}$, could be predicted from the ILD psychophysical threshold, $\theta_{\text {ILD }}$ (Figure 9). We found that, for both sound types, the slope of the regression was positive and significantly different from zero $\left(p_{\mathrm{HP}}=0.001\right.$ and $p_{\mathrm{LP}}=0.046$, for $\mathrm{HP}$ and $\mathrm{LP}$ sounds, respectively). Although the coefficients of determination were not high $\left(r_{\mathrm{HP}}^{2}=0.4\right.$ and $\left.r_{\mathrm{LP}}^{2}=0.2\right)$, it is a considerable correlation given that both parameters are from different fitting models. Furthermore, the fitting direction was as expected as well as a higher correlation for HP, where the cue sensitivity is better defined.

\section{DISCUSSION}

As shown in the presented study, many bilateral CI users have a remarkably good localization performance, which is mainly attributed to adequate ILD processing. However, we also provided evidence that some listeners may have had access to rudimentary ITD information with but also without the FSP strategy. Furthermore, there was a large variability in performance, which so far remains unexplained. Based on our results, we argue that our data suggest that CI users may learn to successfully integrate even rudimentary binaural information.

\section{ILD and ITD Perception}

The bilateral CI listeners in our study were mostly sensitive to ILDs (Figure 4A) since all demonstrated a good sensitivity to cues for high-frequency ILDs. Furthermore, our results show that some listeners had a weak ITD perception within the physiological range (as would be generated in the free field). Some CI listeners with FSP encoding demonstrated a monotonic stimulus-response relation for LP sounds (e.g., Figures 5L,O). This suggests that exposure to weakly informative but robust and consistent cues in everyday life might prompt $\mathrm{CI}$ listeners to learn
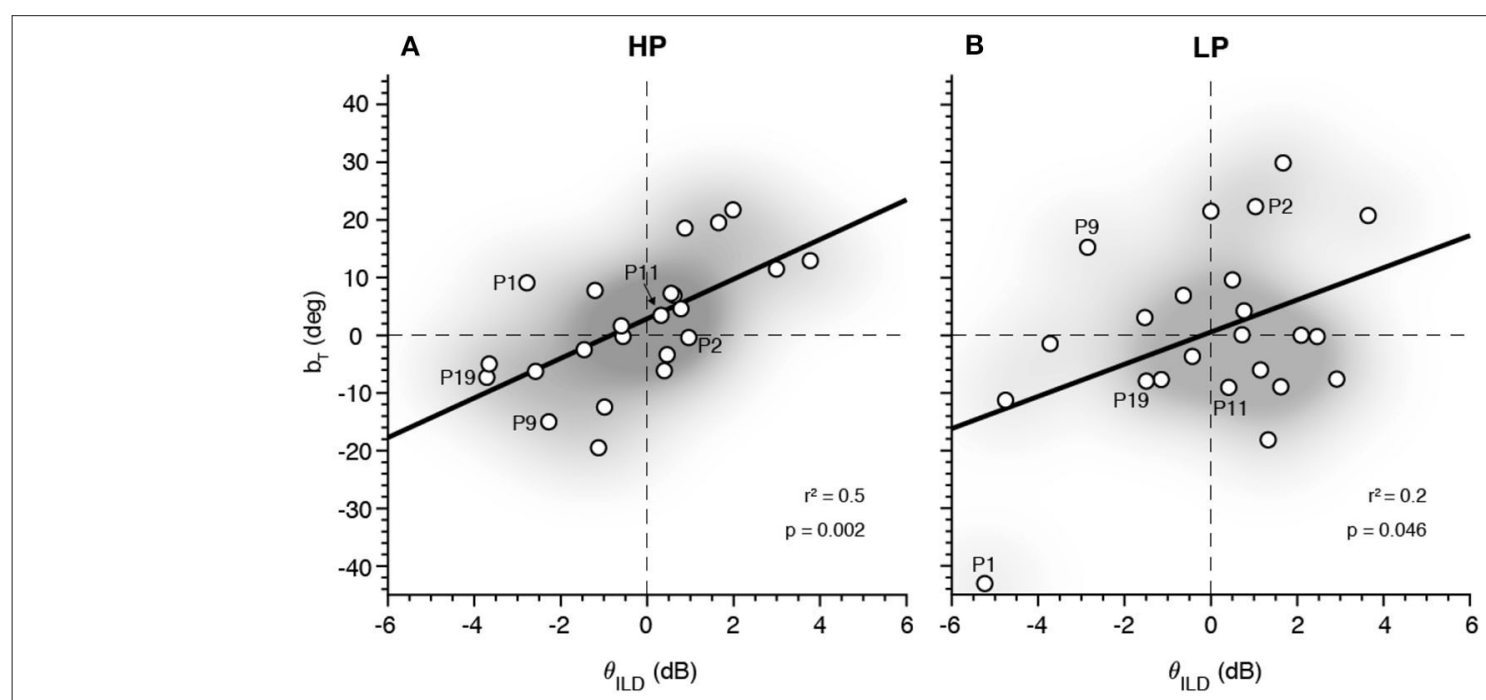

FIGURE 9 | Relation between the interaural level difference (ILD) $\left(\theta_{\| L D}\right)$ and the target sound localization bias (bT) for high-pass (HP) (A) and low-pass (LP) (B). The distribution density of the data points is illustrated with the shadowed gray area. 
to localize even low-frequency sounds (26). Our data support the recent study by Elköf and Tidelhom (21), who reported that half of their FSP population had ITD perception within the physiological range. The participants in their group were all of young age ( $8-13$ years) and had been implanted bilaterally prior to their third year of age. As the brain is most plastic during the early stages in life (27-29), these young listeners may have been able to exploit the information provided from FSP stimulation and use the ITDs also in free-field listening. In the present study, only two of the adult listeners (22 years each) had been implanted at a young age (P8 and P9: 1 year old on the right side), but with a 4-year implantation gap for the second, left ear, and without the FSP encoding protocol in their devices.

However, several studies have demonstrated a life-long plasticity in the human auditory system, which might help CI listeners with post-lingual deafness. Yet, the localization cues provided by the CIs should be unique and consistent for any source location as inconsistent and ambiguous cues cannot induce successful perceptual learning (26, 30-34). The observation that some of the FSP listeners had learned to exploit the subtle and poor ITD cues provided by the FSP encoding protocol may be a promising approach for future improvements.

Lack of ITD perception may also be related to the neural health of the cochlea. In other words, potentially, ITD perception may be reached in CI users with substantial neural substrate of the cochlear and spiral ganglion at the lower-frequency region. In addition, ITD perception may also be influenced by auditory central processing capabilities. In the presence of residual hearing at lower frequencies, the residual function of the peripheral neural substrate can be assessed objectively by measuring auditory steady-state response (ASSR) on each side, and central processing skills may be assessed using binaural masking level difference (BMLD) as an objective measure of binaural cue integration. Due to the lack of residual hearing in our subjects, these measurements would not have been possible to perform in this study group. However, since at present candidates often have considerable residual hearing preimplantation, future prospective studies may include psychoacoustic and objective measures to assess the auditory pathway prior to bilateral implantation. This would expand insights into the variables that determine spatial hearing.

\section{Sound Localization}

The overall sound localization performance is in accordance with other results reported for bilateral CI listeners $(1,5,6,20)$, albeit that accuracy and precision remained worse than that of normal-hearing listeners (Figure 8). Furthermore, we observed some differences in the free-field sound localization performance between FS and no-FS listeners as 11 out of 19 FS listeners had localization gains $<4$ for low-pass sounds (Figure 6C). However, this evidence is not strong enough to support the hypothesis that FS listeners have better localization at a group level.

The deprived ITD sensitivity and the poor ILD representation might underlie this impoverished sound localization performance. Moreover, the weakness of the low-frequency free-field cues seems to be reflected in spatially compressed localization responses $\left(\Delta_{\mathrm{LOC}}<180^{\circ}\right.$; Figure $\left.6 \mathrm{C}\right)$. Still, we observed a very good sound localization performance for LP stimuli in some participants (e.g., P11; Figure 5L). However, considering the MAE values of each group and each frequency band, no significant difference was found (Figure 8).

\section{The Effect of Cl Asymmetry}

In bilateral CI users, asymmetry is quite common due to a mismatch of the bilateral electrode's position or a difference with regard to the time of implantation between the two sides. While one might argue that these asymmetrical factors might affect the listener's spatial hearing performance, our data did not show any strong supportive correlation for this association. Although the exact position of the electrode array and its insertion angle was not measured, since the same electrode design and length were used on each side and with reported full insertion of all electrodes, a comparable insertion angle between both ears is assumed. However, the variation in response bias for the ILD task and the sound localization task might indicate that bilateral CI fitting was not always well-balanced between ears (Figures 4, 7). Typically, each side was fitted independently, and the perception of equal loudness between sides was subjectively assessed in the clinic. However, a subjective procedure may have led to idiosyncratic unbalanced hearing shown in the free-field sound localization results as idiosyncratic non-zero bias. Up to now, there is no standardized protocol in the CI fitting software that helps audiologists to correctly balance each ear according to the subject's perception. In our study, we see that a simple ILD task with a 2 AFC design (left/right response) could be a good initial predictor of the free-field localization bias. However, even with perfectly balanced CIs, sounds might still not be fused as one single source due to device- and patient-related issues (35). Potential asymmetry between left/right electrodes, as well as between-ear frequency allocation tables could also perturb the frequency-specific binaural integration channels $(36,37)$.

Clearly, one might not expect that CI listeners, equipped with a restricted number of frequency channels and a highly limited dynamic range, can approach similar spatial resolution and localization performance as normal-hearing listeners, who can precisely process the encoded information from over 3,000 channels over a huge dynamic range. Yet, binaural integration in bilateral CI recipients might be further improved in the future with optimized bilateral encoding strategies that allow a better synchronization between the two devices, an optimized spectral overlap, and a reliably balanced loudness perception. Moreover, training for spatial hearing is normally not part of CI standard of care, but should be considered as this rehabilitation approach might improve spatial hearing skills in CI users. This way, CI users may truly exploit spatial auditory cues and might map them into a veridical representation of the acoustic environment.

\section{DATA AVAILABILITY STATEMENT}

The raw data supporting the conclusions of this article will be made available by the authors, without undue reservation.

\section{ETHICS STATEMENT}

The studies involving human participants were reviewed and approved by ENT clinic of St. Elisabeth-Hospital of the 
Ruhr-University in Bochum, Germany. The patients/participants provided their written informed consent to participate in this study.

\section{AUTHOR CONTRIBUTIONS}

SA, MA, and SB designed and performed the experiments. SA analyzed the data and wrote the paper. $\mathrm{AE}, \mathrm{CV}, \mathrm{SB}, \mathrm{SD}$, and JT supported the data collection and provided critical revision of the paper. AV, AS, MA, and EM supervised the findings and wrote the final manuscript. EM was the initiator of this collaborative study. All authors contributed to the article and approved the submitted version.

\section{REFERENCES}

1. Nopp P, Schleich P, D'Haese P. Sound localization in bilateral users of MED-EL COMBI 40/40+ cochlear implants. Ear Hear. (2004) 25:205-14. doi: 10.1097/01.AUD.0000130793.20444.50

2. Schleich P, Nopp P, D'Haese P. Head shadow, squelch, and summation effects in bilateral users of the MED-EL COMBI 40/40+ cochlear implant. Ear Hear. (2004) 25:197-204. doi: 10.1097/01.AUD.0000130792.43315.97

3. Van Hoesel RJM. Exploring the benefits of bilateral cochlear implants. Audiol Neuro Otol. (2004) 9:234-46. doi: 10.1159/000078393

4. Litovsky RY, Parkinson A, Arcaroli J, Sammeth C. Simultaneous bilateral cochlear implantation in adults: a multicenter clinical study. Ear Hear. (2006) 27:714-31. doi: 10.1097/01.aud.0000246816.50820.42

5. Litovsky RY, Parkinson A, Arcaroli J. Spatial hearing and speech intelligibility in bilateral cochlear implant users. Ear Hear. (2009) 30:419-31. doi: 10.1097/AUD.0b013e3181a165be

6. Grantham DW, Ashmead DH, Ricketts TA, Labadie RF, Haynes DS. Horizontal-plane localization of noise and speech signals by postlingually deafened adults fitted with bilateral cochlear implants. Ear Hear. (2007) 28:524-41. doi: 10.1097/AUD.0b013e31806dc21a

7. Ausili SA, Backus B, Agterberg $\mathrm{MJH}$, van Opstal AJ, van Wanrooij MM. Sound localization in real-time vocoded cochlear-implant simulations with normal-hearing listeners. Trends Hear. (2019) 23:2331216519847332. doi: $10.1177 / 2331216519847332$

8. Lopez-Poveda EA, Eustaquio-Martín A, Fumero MJ, Stohl JS, Schatzer R, Nopp P, et al. Lateralization of virtual sound sources with a binaural cochlearimplant sound coding strategy inspired by the medial olivocochlear reflex. Hear Res. (2019) 379:103-16. doi: 10.1016/j.heares.2019.05.004

9. Wightman FL, Kistler DJ. The dominant role of low-frequency interaural time differences in sound localization. J Acoust Soc Am. (1992) 91:1648-61. doi: $10.1121 / 1.402445$

10. Blauert J. Spatial Hearing. The Psychophysics of Human Sound Localization. 2nd ed. Cambridge, MA: The MIT Press (1997).

11. Wilson BS, Finley CC, Lawson DT, Wolford RD, Eddington DK, Rabinowitz WM. Better speech recognition with cochlear implants. Nature. (1991) 352:236-8. doi: 10.1038/352236a0

12. Punte AK, De Bodt M, Van De Heyning P. Long-term improvement of speech perception with the fine structure processing coding strategy in cochlear implants. ORL. (2014) 76:36-43. doi: 10.1159/000360479

13. Vermeire K, Punte AK, Van De Heyning P. Better speech recognition in noise with the fine structure processing coding strategy. ORL. (2010) 72:305-11. doi: $10.1159 / 000319748$

14. Müller J, Brill S, Hagen R, Moeltner A, Brockmeier S-J, Stark T, et al. Clinical trial results with the med-el fine structure processing coding strategy in experienced cochlear implant users. ORL. (2012) 74:185-98. doi: 10.1159/000337089

15. Riss D, Hamzavi JS, Katzinger M, Baumgartner WD. Effects of fine structure and extended low frequencies in pediatric cochlear implant recipients. Int $J$ Pediatr Otorhinolaryngol. (2011) 75:573-8. doi: 10.1016/j.ijporl.2011.01.022

\section{FUNDING}

This work was supported by the European Union FP7-PEOPLE2013-ITN project HealthPAC, grant 604063 (SA), a grant from the William Demands og Hustra Ida Emilies Fond (MA), the European Union H2020 ERC Advanced Grant, Project Orient, grant 693400 (AV and AS), and MED-EL.

\section{ACKNOWLEDGMENTS}

We thank Günter Windau, Stijn Martens, and Ruurd Lof for their valuable technical assistance. We are also grateful to Martin Wozniak and Florian Krieger for their valuable assistance and help on part of the data collection.

16. Lorens A, Zgoda M, Obrycka A, Skarzynski H. Fine structure processing improves speech perception as well as objective and subjective benefits in pediatric MED-EL COMBI 40+ users. Int J Pediatr Otorhinolaryngol. (2010) 74:1372-8. doi: 10.1016/j.ijporl.2010.09.005

17. Qi B, Lie Z, Gu X, Liu B. Speech recognition outcomes in Mandarin-speaking cochlear implant users with fine structure processing. Acta Oto Laryngol. (2017) 137:286-92. doi: 10.1080/00016489.2016.1230680

18. Qi B, Krenmayr A, Zhang N, Dong R, Chen X, Schatzer R, et al. Effects of temporal fine structure stimulation on Mandarin speech recognition in cochlear implant users. Acta Oto Laryngol. (2012) 132:1183-91. doi: 10.3109/00016489.2012.699194

19. Chen X, Liu B, Liu S, Mo L, Li Y, Kong Y, et al. Cochlear implants with fine structure processing improve speech and tone perception in Mandarin-speaking adults. Acta Oto Laryngol. (2013) 133:733-8. doi: 10.3109/00016489.2013.773595

20. Dorman MF, Loiselle L, Stohl J, Yost WA, Spahr A, Brown C, et al. Interaural level differences and sound source localization for bilateral cochlear implant patients. Ear Hear. (2014) 35:633-40. doi: 10.1097/AUD.00000000000 00057

21. Eklöf M, Tideholm B. The choice of stimulation strategy affects the ability to detect pure tone inter-aural time differences in children with early bilateral cochlear implantation. Acta Oto Laryngol. (2018) 138:554-61. doi: 10.1080/00016489.2018.1424999

22. Zirn S, Arndt S, Aschendorff A, Laszig R, Wesarg T. Perception of interaural phase differences with envelope and fine structure coding strategies in bilateral cochlear implant users. Trends Hear. (2016) 20:1-12. doi: $10.1177 / 2331216516665608$

23. Kuss M, Jäkel F, Wichmann FA. Bayesian inference for psychometric functions. J Vision. (2005) 5:478-92. doi: 10.1167/5.5.8

24. Kayser H, Ewert SD, Anemüller J, Rohdenburg T, Hohmann V, Kollmeier B. Database of multichannel in-ear and behind-the-ear head-related and binaural room impulse responses. EURASIP J Adv Signal Process. (2009) 2009:298605. doi: 10.1155/2009/298605

25. Van Bentum GC, Van Opstal AJ, Van Aartrijk CMM, van Wanrooji M. Level-weighted averaging in elevation to synchronous amplitude-modulated sounds. J Acoust Soc Am. (2017) 142:3094-103. doi: 10.1121/1.5011182

26. Zonooz B, Arani E, Van Opstal AJ. Learning to localise weakly-informative sound spectra with and without feedback. Sci Rep. (2018) 8:17933. doi: $10.1038 / \mathrm{s} 41598-018-36422-\mathrm{z}$

27. Dennis M. Margaret Kennard (1899-1975): not a 'principle' of brain plasticity but a founding mother of developmental neuropsychology. Cortex. (2009) 46:1043-59. doi: 10.1016/j.cortex.2009.10.008

28. Gutchess A. Plasticity of the aging brain: new directions in cognitive neuroscience. Science. (2014) 346:579-82. doi: 10.1126/science.1254604

29. Kral A, Dorman MF, Wilson BS. Neuronal development of hearing and language: cochlear implants and critical periods. Annu Rev Neurosci. (2019) 8:47-65. doi: 10.1146/annurev-neuro-080317-061513

30. Hofman PM, Van Riswick JGA, Van Opstal AJ. Relearning sound localization with new ears. Nat Neurosci. (1998) 1:417-21. doi: 10.1038/1633 
31. Hofman PM, Vlaming MSMG, Termeer PJJ, van Opstal AJ. A method to induce swapped binaural hearing. J Neurosci Methods. (2002) 113:167-79. doi: 10.1016/S0165-0270(01)00490-3

32. Van Wanrooij MM, Van Opstal AJ. Relearning sound localization with a new ear. J Neurosci. (2005) 25:5413-24. doi: 10.1523/JNEUROSCI.0850-0 5.2005

33. Amitay S, Irwin A, Moore DR. Discrimination learning induced by training with identical stimuli. Nat Neurosci. (2006) 9:1446-8. doi: 10.1038/nn1787

34. Otte RJ, Agterberg MJH, Van Wanrooij MM, Snik AFM, Van Opstal AJ. Agerelated hearing loss and ear morphology affect vertical but not horizontal sound-localization performance. J Assoc Res Otolaryngol. (2013) 14:261-73. doi: 10.1007/s10162-012-0367-7

35. Fitzgerald MB, Kan A, Goupell MJ. Bilateral loudness balancing and distorted spatial perception in recipients of bilateral cochlear implants. Ear Hear. (2015) 36:e225-36. doi: 10.1097/AUD.0000000000000174

36. Kan A, Stoelb C, Litovsky RY, Goupell MJ. Effect of mismatched place-ofstimulation on binaural fusion and lateralization in bilateral cochlear-implant users. J Acoust Soc Am. (2013) 134:2923-36. doi: 10.1121/1.4820889
37. Steel MM, Papsin BC, Gordon KA, Guzman S, Miyasaki G. Binaural fusion and listening effort in children who use bilateral cochlear implants: a psychoacoustic and pupillometric study. PLOS ONE. (2015) 10:e0117611. doi: 10.1371/journal.pone.0117611

Conflict of Interest: SB is an employee of MED-EL.

The remaining authors declare that the research was conducted in the absence of any commercial or financial relationships that could be construed as a potential conflict of interest.

Copyright (C) 2020 Ausili, Agterberg, Engel, Voelter, Thomas, Brill, Snik, Dazert, Van Opstal and Mylanus. This is an open-access article distributed under the terms of the Creative Commons Attribution License (CC BY). The use, distribution or reproduction in other forums is permitted, provided the original author(s) and the copyright owner(s) are credited and that the original publication in this journal is cited, in accordance with accepted academic practice. No use, distribution or reproduction is permitted which does not comply with these terms. 